\title{
DESENVOLVIMENTO E AVALIAÇ̃o DAS CARACTERÍSTICAS FíSICO- QUÍMICAS E DA ACEITAÇÃO SENSORIAL DE DOCE EM MASSA DE CUPUAÇU
}

\author{
JULIETH DAIANE MARQUES DIAS* \\ VIRGINIA KELLY GONÇALVES ABREU** \\ ANA LUCIA FERNANDES PEREIRA** \\ TATIANA DE OLIVEIRA LEMOS*** \\ LEONARDO HUNALDO DOS SANTOS**** \\ VIRLANE KELLY LIMA DA SILVA** \\ ANTONIA SILMARA DE BRITO MOTA*
}

\begin{abstract}
O cupuaçu vem despertando interesse no mercado nacional e internacional, sendo diversos os produtos que podem ser obtidos dessa matéria-prima. Este trabalho teve como objetivo testar formulações de doce em massa de cupuaçu variando a proporção polpa/sacarose, avaliando suas características físico-químicas e aceitação sensorial para a escolha da melhor formulação. Para isso, foram elaboradas as seguintes formulações: F1 $(50 \%$ de polpa e $50 \%$ de sacarose), F2 (55\% de polpa e $45 \%$ de sacarose) e F3 (60\% de polpa e $40 \%$ de sacarose). A quantidade de pectina adicionada correspondeu a $1 \%$ do teor de sacarose. A mistura foi submetida à cocção até atingir concentração de $73^{\circ}$ Brix. Foram realizadas análises de $\mathrm{pH}$, acidez total titulável, cor e atividade de água. A avaliação sensorial foi realizada com 60 provadores não treinados, mediante escala hedônica e intenção de compra. De acordo com os resultados, quanto às características físico-químicas, as formulações atenderam aos padrões legais e tecnológicos para doce em massa e apresentaram valores satisfatórios para cor e atividade de água. Os resultados do teste de aceitação sensorial indicaram não haver diferença significativa entre as formulações em relação aos atributos sabor, doçura e acidez. F3 apresentou maior aceitação $(p<0,05)$ quanto à cor e junto com $F 1$ apresentou maior aceitação $(p<0,05)$ para aparência, textura, impressão global e melhor desempenho quanto à intenção de compra. Desta forma, F1 foi selecionada para a elaboração de doce em massa de cupuaçu, devido menor percentual de polpa que representa vantagem do ponto de vista econômico para a indústria.
\end{abstract}

PALAVRAS-CHAVE: ESCALA HEDONICA; ATIVIDADE DE ÁGUA; COR; PH; DOCE DE FRUTA.

* Graduadas em Engenharia de Alimentos, Universidade Federal do Maranhão, Centro de Ciências Sociais, Saúde e Tecnologia, Campus Avançado de Imperatriz, Av. da Universidade, s/n, Bom Jesus, CEP 65900000, Imperatriz-MA (julieth_daiane@hotmail.com, silmarabrito19@gmail.com).

**Doutoras em Ciência e Tecnologia de Alimentos, Curso de Engenharia de Alimentos, Universidade Federal do Maranhão, Centro de Ciências Sociais, Saúde e Tecnologia, Campus Avançado de Imperatriz, Av. da Universidade, s/n, Bom Jesus, CEP 65900-000, Imperatriz-MA (vkellyabreu@gmail.com; anafernandesp@ yahoo.com.br, virlane.kelly@ufma.br).

*** Doutora em Biotecnologia, Curso de Engenharia de Alimentos, Universidade Federal do Maranhão, Centro de Ciências Sociais, Saúde e Tecnologia, Campus Avançado de Imperatriz, Av. da Universidade, s/n, Bom Jesus, CEP 65900-000, Imperatriz-MA (tharta@bol.com.br).

**** Doutor em Zootecnia, Curso de Licenciatura em Ciências Naturais/ Biologia, Centro de Ciências Sociais, Saúde e Tecnologia, Campus Avançado de Imperatriz, Av. da Universidade, s/n, Bom Jesus, CEP 65900000, Imperatriz-MA (leohunaldo@gmail.com). 


\section{INTRODUÇÃO}

A Região Amazônica possui uma variedade de frutas consideradas potenciais e dentre elas se destaca o cupuaçu (Theobroma grandiflorum Schum), em virtude de seu elevado potencial econômico e valor nutricional. Esta espécie é encontrada espontaneamente nas áreas de mata do sul e nordeste da Amazônia oriental brasileira e nordeste do Maranhão, e também na região Amazônica de países vizinhos (SILVA; SILVA; PENA, 2008; SCHWAN, 2000).

A polpa de cupuaçu tem coloração branco-amarelada e possui alto valor nutricional, sendo fonte de ácido ascórbico (96-111 mg/ 100 g) e compostos fenólicos (20,5 mg/ 100 g). Dessa forma, efeitos benéficos à saúde têm sido atribuídos para o cupuaçu, tendo sua capacidade antioxidante sido bastante estudada (PINENT et al., 2015). A polpa apresenta uma considerável atividade antioxidante (1,7-2,0 $\mu \mathrm{M}$ Trolox/g), sendo esse valor superior a, por exemplo, morango e semelhante a outras frutas nativas brasileiras como a aracá-boi (Eugenia stipitata Mc. Vaugh) e jaracatiá (Jaracatia spinosa Aubli) (KUSKOSKI et al., 2005; PUGLIESE et al., 2013).

Além disso, o cupuaçu é uma fonte de fibra alimentar, principalmente fibra solúvel (SALGADO et al., 2011). A polpa de cupuaçu tem uma composição química que também inclui quantidade considerável de amido, bem como de pectina (VRIESMANN; PETKOWICZ, 2009), que pode fornecer uma textura diferente aos produtos em que são adicionados quando comparada a outras polpas de frutas.

Apesar de suas características nutricionais, a principal forma de comercialização do cupuaçu ainda é como polpa pasteurizada/congelada, em embalagens de polietileno de $200 \mathrm{~g} \mathrm{e}$ $1 \mathrm{~kg}$, e em tambores de $200 \mathrm{~L}$. As duas primeiras formas são utilizadas para a comercialização no estado do Pará (principal produtor do fruto) e a última, quando o produto é comercializado para fora das fronteiras do estado. A polpa pode ser consumida na forma de sucos, néctares, geléias, doces, cremes, tortas, sorvetes, balas, bombons, licores, polpa seca, polpa granulada, entre outras formas (FERREIRA; GUIMARÃES; MAIA, 2008; SILVA; SILVA; PENA, 2008).

Como a maioria das frutas tropicais, o cupuaçu é produzido na região amazônica em período de safra (novembro a julho) e, devido à sua alta perecibilidade, deteriora-se rapidamente, perdendo em torno de 40\% da produção (LOPES; SILVA, 1998; SECRETARIA DE EDUCAÇÃO PROFISSIONAL E TECNOLOGICA, 2007). Diante disso, faz-se necessário disponibilizar opções para a conservação do cupuaçu que associem simplicidade tecnológica, baixos custos de embalagem e acondicionamento, facilidade de transporte e, ainda, boa aceitação no mercado (PEREIRA; ABREU; RODRIGUES, 2018).

Desta forma, a produção de doces é uma forma viável de conservação das frutas, propiciando um melhor aproveitamento das mesmas ao contornar problemas de perecibilidade e de sazonalidade. Estes produtos fazem parte do mercado de confeitaria e são apreciados por um grupo grande e heterogêneo de consumidores: de crianças a idosos. Assim, a produção de um doce a partir do cupuaçu seria uma alternativa para os consumidores obterem os efeitos benéficos provenientes desse fruto (SANTANA; OLIVEIRA, 2005; CAPPA; LAVELLI; MARIOTTI, 2015).

De acordo com Martins et al. (2007), os doces em massa são resultantes do processamento adequado das partes comestíveis das frutas adicionados de açúcares, água, pectina ( 0,5 a 1,5\%) e ajustador de $\mathrm{pH}(3$ a 3,4$)$, além de outros ingredientes e aditivos permitidos pela legislação, até alcançar consistência adequada. A concentração do doce até o teor de sólidos solúveis ( ${ }^{\circ B}$ rix) desejado é realizada através da cocção sob fervura, que também tem como finalidade auxiliar na dissolução do açúcar no suco e a sua interação com a pectina e o ácido para formar o gel. Durante a cocção são também inativados os microorganismos e as enzimas presentes, dando melhores condições de conservação ao produto. Entre os fatores importantes no estudo de produção de doces, destaca-se a importância da relação polpa-sacarose, o tipo de açúcar e a temperatura de cocção (DIAS et al., 2011; JACQUES et al., 2009). Dias et al. (2011) reportaram que a menor concentração de maracujá na elaboração de doces proporcionou maior aceitação sensorial, com destaque para os atributos cor e textura. 
Os doces de cupuaçu encontrados no mercado são em geral produzidos artesanalmente, não apresentando padrão em relação a textura, cor, aroma e sabor, mesmo entre produtos de um mesmo fornecedor. De acordo com Freitas et al. (2012), é necessário melhorar a maneira de produzir os doces de frutas, de forma que atendam às necessidades dos consumidores, que buscam cada vez mais qualidade sensorial, praticidade e conveniência. Assim, a falta de padrão aliada a uma escassez de publicações a respeito de doce em massa de cupuaçu motiva pesquisas para o desenvolvimento de um produto que atenda às necessidades econômicas do produtor e satisfaça o consumidor quanto às características sensoriais. Diante disso, o objetivo do presente trabalho é obter a formulação adequada do doce de cupuaçu com base na avaliação das características físicoquímicas e sensoriais.

\section{MATERIAL E MÉTODOS}

\subsection{MATÉRIA-PRIMA}

Para a obtenção do doce de cupuaçu, foram utilizadas polpas de cupuaçu comerciais pasteurizadas, sacarose comercial e pectina.

\subsection{PROCESSAMENTO DO DOCE EM MASSA DE CUPUAÇU}

Foram elaboradas em triplicata três formulações de doce em massa de cupuaçu. Os doces foram processados em tacho aberto de aço inoxidável com agitação contínua, de acordo com as seguintes formulações: F1 (50\% de polpa de cupuaçu e $50 \%$ de sacarose), F2 (55\% de polpa de cupuaçu e $45 \%$ de sacarose) e F3 (60\% de polpa de cupuaçu e $40 \%$ de sacarose). Todas as formulações foram adicionadas de $1 \%$ de pectina de alto grau de metoxilação, a qual foi acrescentada em relação ao teor de sacarose. $O$ processo foi concluído quando o doce atingiu concentração de sólidos solúveis de aproximadamente $73^{\circ}$ Brix (MENEZES et al., 2009). Os doces foram envasados a quente em embalagens de polipropileno $(300 \mathrm{~g})$ e armazenados a temperatura ambiente $\left(25^{\circ} \mathrm{C}\right)$ até o momento das análises.

Cada formulação foi submetida às análises físico-químicas e avaliação sensorial.

\subsection{ANÁLISES FÍSICO-QUÍMICAS DO DOCE EM MASSA DE CUPUAÇU}

$\mathrm{O} \mathrm{pH}$ das formulações de doce em massa de cupuaçu foi medido em triplicata utilizando pHmetro, calibrado com as soluções tampões de 4 e 7 (INSTRUTHERM, RS 232). Para isso, $10 \mathrm{~g}$ de doce foram dissolvidos em $100 \mathrm{~mL}$ de água destilada e, logo após, foi realizada a leitura.

A acidez total titulável foi determinada por método titulométrico com solução de hidróxido de sódio $(0,1 \mathrm{M})$, usando fenolftaleína como indicador (INSTITUTO ADOLFO LUTZ, 2008). Para a realização, $10 \mathrm{~g}$ das amostras foram dissolvidos em $100 \mathrm{~mL}$ de água e a titulação realizada após inclusão do indicador. Os resultados foram expressos em grama (g) de ácido cítrico/100 g de doce em massa de cupuaçu. A determinação de sólidos solúveis totais foi realizada em refratômetro de bancada (NOVA, 2WA) com escala de 0 a $45^{\circ}$ Brix, de acordo com metodologia descrita pelo Instituto Adolfo Lutz (2008). Os resultados foram expressos em ${ }^{\circ}$ Brix.

A medição objetiva da cor das amostras foi realizada utilizando colorímetro Minolta CR300, Tokyo, operando no sistema $\operatorname{CIE}\left(L^{*}, a^{*} e b^{*}\right)$. Sendo $L^{*}$ a luminosidade, variando de 0 (preto) para 100 (branco); $a^{*}$ a intensidade da cor vermelha, variando de verde (-60) a vermelho (+60); e b* a intensidade da cor amarela, variando de azul (-60) a amarelo (+60). A calibração do aparelho foi realizada por meio de placa de cerâmica branca, utilizando-se o iluminante $D_{65}$. $A$ atividade de água foi realizada a $25{ }^{\circ} \mathrm{C}$, por medida direta nas amostras de doce em massa usando equipamento digital (Aqualab®, 4TE). 


\subsection{ACEITAÇÃO SENSORIAL DO DOCE EM MASSA DE CUPUAÇU}

Este trabalho foi submetido e aprovado pelo Comitê de Ética em pesquisa da Universidade Federal do Maranhão (CAAE: 16726213.2.0000.5087). Todos os participantes assinaram o termo de Consentimento Livre Esclarecido, seguindo as normas do Conselho Nacional de Ética em Pesquisa com Humanos.

Os testes sensoriais foram realizados no Laboratório de Análise Sensorial da Universidade Federal do Maranhão. Participaram da avaliação sensorial 60 julgadores não treinados de ambos os sexos ( $72 \%$ mulheres e $28 \%$ homens), entre alunos e funcionários da Universidade Federal do Maranhão com faixa etária predominante entre 18 e 25 anos (81\%). O teste foi conduzido em cabines individuais com incidência de luz branca, sob condições controladas. Amostras (aproximadamente $15 \mathrm{~g})$ foram servidas à temperatura ambiente $\left(25^{\circ} \mathrm{C}\right)$ em copos plásticos descartáveis de $50 \mathrm{~mL}$ codificados com três dígitos aleatórios, de forma monádica sequencial, seguindo-se delineamento de blocos completos balanceados com relação à ordem de apresentação.

Avaliou-se a aceitação sensorial dos doces em massa utilizando Escala Hedônica estruturada mista de 9 pontos ( 9 = gostei muitíssimo, 5 = não gostei, nem desgostei; 1 = desgostei muitíssimo), mediante os atributos: cor, aparência, sabor, doçura, acidez, textura e aceitação global (STONE; SIDEL; SCHUTZ, 2004).

A intenção de compra do produto foi avaliada através da impressão global dos consumidores com Escala de Atitude de Compra estruturada mista de 5 pontos ( 5 = certamente compraria; 3 = tenho dúvidas se compraria; 1 = certamente não compraria) (MEILGAARD; CIVILLE; CARR, 1991).

Para o cálculo do Índice de Aceitabilidade (IA) de cada formulação, foi utilizada a seguinte expressão: $I A(\%)=A X 100 / B$, em que $\mathrm{A}=$ nota média obtida para o produto e $\mathrm{B}=$ nota máxima dada ao produto (TEIXEIRA; MEINERT; BARBETTA, 1987).

\subsection{ANÁLISE ESTATISTICA}

Os resultados das análises físico-químicas foram obtidos através de médias de três repetições e desvio padrão dos valores encontrados e os dados foram tabulados no Excel 2010.

Para os dados da avaliação sensorial, foi considerado um experimento em blocos casualizados para avaliar três formulações de doce de cupuaçu (tratamentos) quanto aos atributos: cor, aparência, sabor, doçura, acidez, textura, impressão global e intenção de compra. Na presente análise, os provadores foram considerados os blocos.

Por se tratar de variáveis quantitativas discretas, os tratamentos foram avaliados quanto às variáveis citadas utilizando o teste não paramétrico de Friedman (mais de duas amostras dependentes) a $5 \%$ de significância, em que não há suposições sobre a distribuição dos dados, como descrito em Gibbons e Chakraborti (2010). As variáveis significativamente diferentes entre as amostras seguiram para o teste de comparação múltipla de Friedman a $5 \%$ de significância. Todos os dados foram tabulados no Excel 2010 e os testes realizados no programa SAS (SAS, 2000).

\section{RESULTADOS E DISCUSSÃO}

\subsection{ANÁLISES FÍSICO-QUÍMICAS DO DOCE EM MASSA DE CUPUAÇU}

Os resultados das análise físico-químicas das formulações de doce em massa de cupuaçu são apresentados na Tabela 1. Os valores de $\mathrm{pH}$ das formulações do doce em massa variaram entre 3,20 e 3,25. Para acidez total titulável, o doce em massa de cupuaçu apresentou valores que variaram de 1,20 a $1,40 \mathrm{~g}$ de ácido cítrico/100 g. A medição instrumental da cor mostrou valores de $\mathrm{L}^{*}$ (luminosidade) entre 40,76 e 46,86. Os valores de $a^{*}$ (intensidade de vermelho) e b* (intensidade de 
amarelo), variaram de $-3,07$ a -1,98 e de 14,80 a 22,38, respectivamente. Quanto à de atividade de água $(A w)$, os valores encontrados para as três formulações variaram entre 0,71 e 0,76 (TABELA 1).

\section{TABELA 1 - VALORES MÉDIOS E DESVIO PADRÃO PARA ANÁLISES FÍSICO-QUÍMICAS DA POLPA DE CUPUAÇU E DO DOCE EM MASSA DE CUPUAÇU COM DIFERENTES PROPORÇÕES DE POLPA E SACAROSE}

\begin{tabular}{ccccc}
\hline & Polpa de cupuaçu & F1 & F2 & F3 \\
\hline $\mathbf{p H}$ & $3,32 \pm 0,01$ & $3,25 \pm 0,01$ & $3,22 \pm 0,01$ & $3,20 \pm 0,01$ \\
\hline $\mathbf{A T T}$ & $2,00 \pm 0,05$ & $1,40 \pm 0,01$ & $1,40 \pm 0,01$ & $1,20 \pm 0,01$ \\
\hline $\mathbf{L}^{*}$ & $58,70 \pm 0,77$ & $40,76 \pm 0,61$ & $40,00 \pm 0,20$ & $46,86 \pm 0,01$ \\
\hline $\mathbf{a}^{*}$ & $-7,71 \pm 0,03$ & $-3,07 \pm 0,12$ & $-3,49 \pm 0,19$ & $-1,98 \pm 0,20$ \\
\hline $\mathbf{b}^{*}$ & $23,44 \pm 0,98$ & $14,80 \pm 2,19$ & $10,74 \pm 0,61$ & $22,38 \pm 1,17$ \\
\hline $\mathbf{A w}$ & $0,99 \pm 0,01$ & $0,71 \pm 0,01$ & $0,73 \pm 0,00$ & $0,76 \pm 0,01$ \\
\hline
\end{tabular}

F1=polpa de cupuaçu: sacarose (50:50), F2= polpa de cupuaçu: sacarose $(55: 45), F 3=$ polpa de cupuaçu: sacarose (60:40); ATT = acidez total titulável $\left(\mathrm{g}\right.$ de ácido cítrico/ $100 \mathrm{~g}$ ); $\mathrm{L}^{*}=$ luminosidade; $\mathrm{a}^{*}=$ intensidade de vermelho, $\mathrm{b}^{*}=$ intensidade de

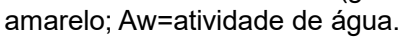

Os resultados de $\mathrm{pH}$ encontrados para o doce em massa de cupuaçu atendem aos padrões tecnológicos para doces em massa, cujo valor ideal, segundo Gava, Silva e Frias (2009), situa-se entre 3,2 e 3,5. Para a produção do doce em massa de cupuaçu não foi necessário adicionar ácido à formulação, uma vez que o fruto possui pH de 3,32 (TABELA 1). Além disso, observou-se que o pH da polpa e do doce foram bem próximos, indicando que o processamento não influenciou esse parâmetro.

Os valores de acidez total titulável obtidos para as formulações de doce em massa de cupuaçu foram altos quando comparados a outros doces de frutas. Dias et al. (2011) reportaram acidez variando de 0,62 a $0,90 \mathrm{~g}$ de ácido cítrico $/ 100 \mathrm{~g}$ em doce massa de albedo do maracujá e Menezes et al. (2009) observaram valores variando de 0,42 a 0,92 g ácido cítrico/ $100 \mathrm{~g}$ em doce de goiaba. Os expressivos valores do presente estudo são resultantes dos altos valores de acidez da polpa de cupuaçu que foi de 2,00 g ácido cítrico/100 g (TABELA 1). Esses valores estão de acordo com a legislação vigente que estabelece teor mínimo permitido para a polpa de cupuaçu de 1,50 expressos em ácido cítrico g/100 g (BRASIL, 2000).

Quanto à cor, houve redução nos valores de $L^{*}$ (luminosidade) e aumento nos valores de $a^{*}$ (intensidade de vermelho) dos doces em massa de cupuaçu quando comparados com os da polpa (TABELA 1). Dias et al. (2011) também reportaram redução na luminosidade e aumento da intensidade de vermelho em doces quando comparados com a matéria-prima, atribuindo tal resultado ao escurecimento dos doces. Faria et al. (2003) afirmaram que, durante o cozimento, ocorre escurecimento devido à oxidação dos pigmentos, principalmente em função das altas temperaturas. Ocorre também um certo grau de caramelização devido ao tempo de cozimento e presença de açúcares redutores (TORREZAN et al., 2000). Desta forma, a redução obtida nesse parâmetro no presente estudo deve-se a alterações proporcionadas na cor em virtude do processamento do doce.

No que se refere ao componente de cor $b^{*}$ (intensidade de amarelo), foi observada uma redução quando comparado com a polpa (TABELA 1). Policarpo et al. (2007) reportaram que a adição de pectina alterou os parâmetros $a^{*} e b^{*}$ das formulações, obtendo-se uma formulação com tendência maior para vermelho e menor para amarelo, diminuindo a cor amarelo-esverdada, 
característica da polpa original. Portanto, o aumento de $a^{*}$ e a redução de $b^{*}$ dos doces em relação à polpa pode ser resultante da pectina adicionada.

A formulação F3 apresentou maior valor de luminosidade e de intensidade de cor amarela, indicando um doce mais claro. Esse resultado pode explicar a melhor aceitação da cor dessa amostra em relação às demais na avaliação sensorial feita pelos julgadores no presente estudo (TABELA 2). Godoy et al. (2008), trabalhando com doce de acerola em massa também observou uma relação entre a medida instrumental da cor e a resposta encontrada na análise sensorial para esse atributo. A cor do doce de acerola em massa elaborado com o genótipo CMF-017 que apresentou a maior intensidade de vermelho $\left(a^{*}\right)$, também obteve melhor aceitação da cor pelos julgadores que as demais formulações.

Os resultados de atividade de água obtido foram similares aos resultados reportados por Menezes et al. (2009) para doce de goiaba com as mesmas proporções polpa/ sacarose. Essa variação na Aw pode ser explicada pelo conteúdo de açúcar presente em cada formulação, em que F1 possui a maior concentração seguida das formulações F2 e F3. De acordo com Menezes et al. (2009), a atividade de água diminuiu com o aumento da adição de açúcar, pois este composto é altamente higroscópico, o que diminui o teor de água livre no alimento, a qual é utilizada para as reações deteriorativas. Torrezan, Jardine e Vitali (1999), estudando o efeito da adição de solutos e ácidos em polpa de goiaba, também verificaram que, quanto maior a adição de sacarose, menor a atividade de água do sistema. Segundo Franco e Landgraf (1996), a atividade de água de doces deve estar entre 0,7 a 0,8 . Assim, as formulações de doce em massa de cupuaçu encontram-se dentro desses limites, garantindo uma boa estabilidade física e microbiológica, evitando o crescimento de microorganismos e reações de escurecimento não enzimático (BELOVIC et al., 2017).

\subsection{ACEITAÇÃO SENSORIAL DO DOCE EM MASSA DE CUPUAÇU}

Os resultados do teste de aceitação sensorial indicaram não haver diferença significativa entre as formulações avaliadas em relação aos atributos sabor, doçura e acidez. Já para os atributos cor, aparência, textura, impressão global e para a atitude de compra houve diferença significativa $(p<0,05)$ entre as formulações de doce de cupuaçu (TABELA 2).

\section{TABELA 2 - VALORES HEDÔNICOS PARA OS ATRIBUTOS SENSORIAIS E A ATITUDE DE COMPRA DE DOCE EM MASSA DE CUPUAÇU}

\begin{tabular}{llll}
\hline & \multicolumn{1}{c}{ F1 } & F2 & F3 \\
\hline Cor & $6,70 \pm 1,73^{\mathrm{b}}$ & $4,58 \pm 2,04^{\mathrm{c}}$ & $7,85 \pm 1,09^{\mathrm{a}}$ \\
\hline Aparência & $7,12 \pm 1,51^{\mathrm{a}}$ & $4,95 \pm 2,00^{\mathrm{b}}$ & $7,83 \pm 0,91^{\mathrm{a}}$ \\
\hline Sabor & $6,75 \pm 1,82^{\mathrm{a}}$ & $6,75 \pm 1,76^{\mathrm{a}}$ & $7,05 \pm 1,51^{\mathrm{a}}$ \\
\hline Doçura & $6,33 \pm 1,93^{\mathrm{a}}$ & $6,38 \pm 1,83^{\mathrm{a}}$ & $6,43 \pm 1,84^{\mathrm{a}}$ \\
\hline Acidez & $6,08 \pm 1,99^{\mathrm{a}}$ & $6,00 \pm 1,94^{\mathrm{a}}$ & $6,23 \pm 1,93^{\mathrm{a}}$ \\
\hline Textura & $7,28 \pm 1,47^{\mathrm{a}}$ & $5,78 \pm 1,90^{\mathrm{b}}$ & $7,70 \pm 1,17^{\mathrm{a}}$ \\
\hline Impressão global & $6,76 \pm 1,78^{\mathrm{a}}$ & $6,02 \pm 1,87^{\mathrm{b}}$ & $7,16 \pm 1,58^{\mathrm{a}}$ \\
\hline Atitude de compra & $3,78 \pm 1,02^{\mathrm{a}}$ & $3,24 \pm 1,18^{\mathrm{b}}$ & $4,05 \pm 1,05^{\mathrm{a}}$
\end{tabular}

Médias com letras diferentes na mesma linha diferem entre si $(p<0,05)$ pelo teste de comparação múltipla de Friedman. F1=polpa de cupuaçu: sacarose (50:50); F2=polpa de cupuaçu: sacarose (55:45); F3=polpa de cupuaçu: sacarose (60:40). 
Quanto ao atributo cor, $F 3(7,85)$ obteve a maior aceitação $(p<0,05)$, sendo seguida das formulações F1 $(6,70)$ e F2 $(4,58)$ (TABELA 2). Essa maior aceitação pode estar relacionada à intensidade de coloração amarela, visto que, pela análise instrumental de cor, a formulação $F 3$ apresentou maior valor do componente $b^{*}$ entre os doces elaborados (TABELA 1).

A aceitação dos atributos aparência, textura, impressão global e a intenção de compra das formulações $\mathrm{F} 1$ e F3 não diferiram significativamente entre si, todavia apresentaram valores superiores $(p<0,05)$ que a formulação F2 (TABELA2). Diferentemente do presente estudo, Figueiredo et al. (2009), ao avaliarem o efeito da adição de suco de maracujá em doce, observaram que a maior concentração do suco melhorou as características de qualidade, como também a aceitação do doce. Assim, o resultado obtido no presente estudo é positivo, uma vez que o decréscimo da proporção de polpa não afetou a aceitação sensorial.

Em relação à aparência, F1 e F3 apresentaram valores variando entre "gostei moderadamente" e "gostei muito", indicando encontrar-se na região de aceitação da escala hedônica. Esse resultado é importante, uma vez que o aspecto visual é o primeiro critério adotado pelo consumidor na escolha dos alimentos (ABRAHÃO et al., 2009). Já para F2, os valores médios variaram entre "desgostei ligeiramente" e "nem gostei, nem desgostei", o que evidencia uma rejeição na aparência dessa formulação.

De maneira geral, as médias dos atributos sensoriais das formulações F1 e F3 variaram entre as categorias "gostei moderadamente" e "gostei muito" da escala hedônica, indicando, assim, uma aceitação dessas formulações. Já para F2, as médias variaram entre "desgostei ligeiramente" e "gostei moderadamente. Salienta-se que, para todos os atributos sensoriais, exceto doçura, a F2 obteve menores notas quando comparada com F1 e F3 (TABELA 2).

Para o índice de aceitabilidade das formulações de doce em massa de cupuaçu, foram obtidos valores de 75,$15 ; 66,87$ e 79,56\% para F1, F2 e F3, respectivamente. De acordo com Teixeira, Meinert e Barbetta (1987), o produto pode ser considerado aceito em termos de suas propriedades sensoriais quando alcançar IA de no mínimo $70 \%$. Portanto, pode-se observar que os doces de F1 e F3 foram bem aceitos.

Desta forma, entre as três formulações avaliadas, recomenda-se a formulação F1 por conta do menor percentual de polpa de cupuaçu, o que representa vantagem do ponto de vista econômico. Além disso, não foram evidenciadas diferenças significativas na maioria dos atributos sensoriais, exceto na cor, quando comparados F1 e F3, que foi a formulação com maiores notas em todos os atributos sensoriais.

\section{CONCLUSÃO}

Os resultados deste estudo permitem concluir ser viável a produção de doce em massa de cupuaçu com diferentes proporções de polpa de cupuaçu/sacarose que satisfaça a legislação e seja aceito sensorialmente.

Quanto às características físico-químicas, as formulações testadas atendem aos padrões legais e tecnológicos para doce em massa e apresentam valores satisfatórios para atividade de água e componentes de cor.

As formulações de doce em massa de cupuaçu obtiveram bom desempenho para os atributos sensoriais avaliados e para intenção de compra.

As formulações F1 e F3 não diferem em relação à aceitação para aparência, textura, impressão global e quanto à intenção de compra.

Entre as três formulações avaliadas, recomenda-se a formulação F1 devido ao menor percentual de polpa, que representa vantagem do ponto de vista econômico para a indústria. 


\section{ABSTRACT \\ MARMALADE MADE FROM CUPUASSU: PHYSICAL AND CHEMICAL CHARACTERISTICS AND ACCEPTABILITY}

The interest for cupuassu has increased at national and international markets, with several products that can be obtained from this raw material. The aim of this study was to evaluate formulations of marmalade cupuassu varying the pulp/ sucrose ratio, evaluating their physicochemical characteristics and sensory acceptance in order to choose the best formulation. For this, the following formulations were prepared: F1 ( $50 \%$ pulp and $50 \%$ sucrose), F2 (55\% pulp and $45 \%$ sucrose) and $\mathrm{F} 3$ ( $60 \%$ pulp and $40 \%$ sucrose). The added pectin was $1 \%$ sucrose content. The mixture was coocked to reach the concentration of $73^{\circ} \mathrm{Brix} . \mathrm{pH}$, total acidity, color and water activity analyzes were performed. The sensory evaluation was performed by 60 untrained panellists using hedonic scale and purchase intent. According to the results ofthe physical and chemical characteristics, the formulations are according to legal and technological standards for marmalade and showed satisfactory values for color and water activity. The results of the sensory acceptance evaluation indicated no significant difference between formulations to flavor, sweetness and acidity attributes. F3 showed the highest acceptance $(p<0.05)$ on color and $F 1$ showed a greater acceptance $(p<0.05)$ for appearance, texture, overall impression and better performance for purchase intent. Thus, F1 was selected for the preparation of marmalade cupuassu due to lower percentage of pulp that is advantageous from an economic point of view for the industry.

KEYWORDS: HEDONIC SCALE; WATER ACTIVITY; COLOR; PH; MARMALADE.

\section{REFERÊNCIAS}

1 ABRAHÃO, C.; MIGUEL, A. C. A.; DIAS, J. R. P. S.; SPOTO, M. H. F.; SILVA, P. P. M. Aplicação do método QDF na avaliação do perfil do consumidor de melão amarelo. Ciência e Tecnologia de Alimentos, v.29, n.4, p.716-720, 2009.

2 BELOVIC, M.; TORBICA, A.; PAJIC-LIJAKOVIC, I.; MASTILOVIC, J. Development of low calorie jams with increased content of natural dietary fibre made from tomato pomace. Food chemistry, v.237, p. 1226-1233, 2017.

3 BRASIL. Ministério da Agricultura, Pecuária e Abastecimento/MAPA. Instrução Normativa $n^{\circ} 1$, de 07 de Janeiro de 2000. Regulamento Técnico Geral para fixação dos padrões de identidade e qualidade para polpa de fruta. Diário Oficial da União, Brasília, DF, 10 de Janeiro de 2000, seção 1.

4 CAPPA, C.; LAVELLI, V.; MARIOTTI, M. Fruit candies enriched with grape skin powders: physicochemical properties. LWT - Food Science and Technology, v. 62, p. 569-575, 2015.

5 DIAS, M. V.; FIGUEIREDO, L. P.; VALENTE, W. A.; FERRUA, F. Q.; PEREIRA, P. A. P.; PEREIRA, A. G. T.; BORGES, S. V.; CLEMENTE, P. R. Estudo de variáveis de processamento para produção de doce em massa da casca do maracujá (Passiflora edulis f. flavicarpa). Ciência e Tecnologia de Alimentos, v. 31, n.1, p. 65-71, 2011.

6 FARIA, R.Q.; ANJOS, V.; GREGORIO, S.R.; ENDO, E.; POLICARPO, V.M.N.; BORGES, S.V.; CAVALCANTE, N.B. Efecto de aditivos sobre color, textura y

7 aceptación del dulce de umbu (Spondias tuberosa Arr. Cam.) verde. Alimentaria, v. 346, p. 111-116, 2003.

8 FERREIRA, G. M.; GUIMARÃES, M. J. O. C.; MAIA, M. C. A. Efeito da temperatura e taxa de cisalhamento nas propriedades de escoamento da polpa de cupuaçu ( $T$. grandiflorum schum) integral. Revista Brasileira de Fruticultura, v. 30, n. 2, p.385-389, 2008.

9 FIGUEIREDO, L. F.; VALENTE, W. A.; DIAS, M. V.; BORGES, S. V.; PEREIRA, P. A. P.; PEREIRA, A. G. T.; CLEMENTE, P. R. Efeito da adição de suco de maracujá e tempo de cozimento sobre a qualidade de doces do albedo de maracujá em calda. Ciência e Tecnologia de Alimentos, v. 29, n. 4, p. 840-846, 2009.

FRANCO, B. G. M.; LANGDGRAF, M. Microbiologia dos Alimentos. São Paulo: Atheneu, 1996. 182 p. 
11 FREITAS, M. L. F.; MENEZES, C. C.; CARNEIRO, J. D. S.; REIS, R. P. Diagnóstico do consumo e processo produtivo de doces de frutas produzidos artesanalmente. Alimentos e Nutrição, v. 23, n. 4, p. 585-595, 2012.

12 GAVA, A.J.; SILVA, C. A. B.; FRIAS, J. R. G. Tecnologia de alimentos: princípios e aplicações. São Paulo: Nobel, 2009, $511 p$. GIBBONS, J. D.; CHAKRABORTI, S. Nonparametric Statistical Inference, 5th Edition, CRC Press, Florida, 2010.

14 GODOY, R. C. B.; MATOS, E. L. S.; AMORIM, T. S.; SOUSA NETO, M. A.; RITZINGER, R.; WASZCZYNSKYJ, N. Avaliação de genótipos e variedades de acerola para consumo in natura e para elaboração de doces. Boletim do Centro de Pesquisa e Processamento de Alimentos, v. 26, n. 2, p. 197-204, jul./dez. 2008.

15 INSTITUTO ADOLFO LUTZ . Normas analíticas do Instituto Adolfo Lutz: método químicos e físicos para análise de alimentos. 4. ed. São Paulo: IMESP, 2008. v. 1p. 1020.

16 JACQUES, A. C.; PERTUZATTI, P. B.; BARCIA, M. T.; ZAMBIAZI, R. C. Doce em massa de amora preta (rubus spp): análise sensorial e de fitoquímicos. Alimentos e Nutrição, v.20, n.4, p. 625-631, 2009.

17 KUSKOSKI, E. M.; ASUERO, A. G.; TRONCOSO, A. M.; MANCINI-FILHO, J.; FETT, R.). Aplicación de diversos me todos químicos para determinar' actividad antioxidante em pulpa de frutos. Food Science and Technology, v. 25 , p. 726-732, 2005

18 LOPES, C. M. D. A.; SILVA, N. M. Impacto Econômico da Broca do Cupuaçu, Conotrachelus humeropictus Field (Coleoptera: Curculionidae) nos Estados do Amazonas e Rondônia. Anais da Sociedade Entomológica do Brasil, v. 27, n. 3, p. 481-483, 1998.

19 MARTINS, M. L. A.; BORGES, S. V.; DELIZA, R.; CASTRO, F. T. de; CAVALCANTE, N. B. de. Características de doce em massa de umbu verde e maduro e aceitação pelos consumidores. Pesquisa Agropecuária Brasileira, v.42, n.9, p.1329-1333, 2007

20 MEILGAARD, M.; CIVILLE, G. V.; CARR, B. T. Sensory evaluation techniques. 2 nd ed. Flórida: CRC Press, 1991. $354 \mathrm{p}$.

21 MENEZES, C. C.; BORGES, S. V.; CIRILLO, M. A.; FERRUA, F. Q.; OLIVEIRA, L.F.; MESQUITA, K. S. Caracterização física e físico-química de diferentes formulações de doce de goiaba (Psidium guajava L.) da cultivar Pedro Sato. Ciência e Tecnologia de Alimentos, v. 29, n. 3, p. 618-625, 2009.

22 PEREIRA, A. L. F. P.; ABREU, V. K. G.; RODRIGUES, S. Cupuassu - Theobroma grandiflorum. In: Exotic fruits. London: Academic press, 2018, p. 159-162.

23 PINENT, M.; CASTEll-AUVÍ, A.; GENOVESE, M. I.; SERRANO, J.; CASANOVA, A.; BLAY, M.; ARDÉVOL, A. Antioxidant effects of proanthocyanidin-rich natural extracts from grape seed and cupuassu on gastrointestinal mucosa. Journal of the Science of Food and Agriculture, v. 96, p. 178-182, 2015. PUGLIESE, A. G.; TOMAS-BARBERAN, F. A.; TRUCHADO, P.; GENOVESE, M. J. Flavonoids, proanthocyanidins, vitamin C, and antioxidant activity of Theobroma grandiflorum (cupuassu) pulp and seeds. Journal of Agricultural and Food Chemistry, 61, 2720-2728, 2013.

24 POLICARPO, V.M.N.; BORGES, S.V.; ENDO, E.; CASTRO, F.T.; DAMICO, A.A.; CAVALCANTI, N.B. Estabilidade da cor de doces em massa de polpa de umbu (Spondias TuberosaArr. Cam.) no estádio de maturação verde. Ciência e Agrotecnologia, v. 31, n. 4, p. 1102-1107, 2007.

25 SALGADO, J. M.; RODRIGUES, B. S.; DONADO-PESTANA, C. M.; DOS SANTOS DIAS, C. T.; MORZELLE, M. C. Cupuassu (Theobroma grandiflorum) peel as potential source of dietary fiber and phytochemicals in whole-bread preparations. Plant Foods for Human Nutrition, v. 66, p. 384-390, 2011

SANTANA, A.; OLIVEIRA, L. Aproveitamento da casca de melancia (Curcubita citrullus, Shrad) na produção artesanal de doces alternativos. Alimentos e Nutrição. v. 16, n. 4, p. 363-368, 2005.

27 SAS -STATISTICAL ANALYSIS SYSTEM. SAS software: user's guide. Version 8.2. Cary: 2000. 291p.

28 SCHWAN, R. F. Cupuaçu [Theobroma grandiflorum (Willd Ex Spreng.)]. In: ALVES, R. E., FILGUEIRAS, H.A.C., MOURA, C.F.H. (coords.). Caracterização de frutas nativas da América Latina. Jaboticabal: FUNEP, p.31-34 (Série Frutas Nativas, 9), 2000.

29 SECRETARIA DE EDUCAÇÃO PROFISSIONAL E TECNOLOGICA. Cupuaçu. Ministério da educação, Brasília, 2007, $29 \mathrm{p}$.

30 SILVA, A. E.; SILVA, L. H. M.; PENA, R. S. Comportamento higroscópico do açaí e cupuaçu em pó. Ciência e Tecnologia de Alimentos, v. 28, n.4, p. 895-901, 2008.

31 STONE, H.; SIDEL, J. L.; SCHUTZ, H. G. Sensory Evaluation Practices. 3. ed. Boston: Elsevier, 2004.374 p.

32 TEIXEIRA, E.; MEINERT, E.M.; BARBETTA, P.A. Análise sensorial de alimentos. Florianópolis: UFSC, 1987. p. 60. 
33 TORREZAN, R.; JARDINE, J. G.; VITALI, A. A. Efeito da adição de solutos e ácidos em polpa de goiaba. Ciência e Tecnologia de Alimentos, v. 19, n. 1, p. 43-45, 1999.

34 TORREZAN, R.; FERREIRA, V.L.P.; YOTSUYANAGI, K.; JARDINE, J.G.; VITALI, A.A. Efeito da adição de ingredientes na cor de polpa de goiaba. Boletim do Centro de Pesquisa de Processamento de Alimentos, v. 18, n. 2, p. 209-220, 2000.

35 VRIESMANN, L. C.; PETKOWICZ, C. L. Polysaccharides from the pulp of cupuaçu (Theobroma grandiflorum): Structural characterization of a pectic fraction. Carbohydrate Polymers, v. 77, p. 72-79, 2009 